\title{
Capillary Electrophoresis Method for Determining the Content of Rutin in Fructus Kochiae
}

\author{
Huiqin Wang, Wei Hu, Ting Zhou, Haixing Liua, \\ Chemistry \& Chemical and Environmental Engineering College, Weifang University, Weifang \\ 261061, P.R. China. \\ *, ahaixingliu@tom.com
}

\begin{abstract}
The experiment was Carried out for the determination of rutin content in Fructus Kochiae by high performance capillary electrophoresis method. The borax solution as buffer solution was chosen, and its concentration was $20 \mathrm{mmol} / \mathrm{L}$ at a constant voltage of $20 \mathrm{kV}$ and injecting time of $10 \mathrm{~s}$. The detection wavelength was $254 \mathrm{~nm}$. Measured rutin content in Fructus Kochiae was $0.115 \mathrm{mg} / \mathrm{g}$ $(R S D=10.5 \%)(n=6)$. The recovery was $103.6 \%(R S D=7.2 \%, n=4)$.
\end{abstract}

Keywords: Capillary electrophoresis, Fructus Kochiae, Rutin.

\section{Introduction}

Fructus kochiae is traditional Chinese medicine. It has the effect of Clearing heat, removing dampness, expelling wind and stopping itching. It is used for the symptom of painful urination, itchy skin and so on [1]. It has the function of anti-inflammatory and anti-pathogenic microorganism so on. It is chiefly used in the treatment of skin tinea and eczema disease. Its active ingredient was triterpene, saponin and flavonoids [2-4]. Zeng [5] established a RP-HPLC method for the determining 4'-O- $\beta$-D-glycosyl-5-Omethylvisamminol in Jingfuzhiyang Granules( Herba Schizonepetae, Fructus kochiae, Radix Saposhnikoviae, Fructus Crataegi, etc.). The sample was performed on Turnner Kromasil C18 column $(4.6 \mathrm{~mm} \times 200 \mathrm{~mm}, 5 \mu \mathrm{m})$ with mobile phase of methanol- $0.05 \mathrm{~mol} / \mathrm{L}$ potassium dihydrogen phosphate-acetic acid-isopropyl alcohol (85:170:4:4, v/v) and detection wavelength of $254 \mathrm{~nm}$ and the flow rate of $1.0 \mathrm{~mL} / \mathrm{L}$. The column temperature was $30^{\circ} \mathrm{C}$. Luo et al [6] Established a method for measure Momordin Ic in Fructus Kochiae gel, the HPLC analysis was carried out on a Agilent HC-C18 column $(160 \mathrm{~mm} \times 4.6 \mathrm{~mm}, 5 \mu \mathrm{m})$ with a mobile phase of methanol- $1.3 \%$ acetic acid (85:15). The flow rate was $1.0 \mathrm{~mL} / \mathrm{min}$ and the detection wavelength was $208 \mathrm{~nm}$. Xia et al [7] compared the content of momordin Ic and total saponin in Fructus kochiae from eleven producing areas. The HPLC-ELSD method were adopted for the determining the content of momordin Ic and total saponin, respectively. The content of momordin Ic in Fructus kochiae was related to that of total saponin. Cheng et al [8] investigated the antioxidant activity of the whole herbs of Kochia scoparia and theirs ferulic acid derivatives in vitro through 1,1-diphenyl-2-picryhydrazyl free radical (DPPH) and $\mathrm{Fe}^{3+}$ reduction ability experiments. The antioxidant activity of extracts and ferulic acid derivatives were also compared with vitamin $\mathrm{C}$ and salicylic acid. The results indicated that the antioxidant activity of extracts from the whole herbs of Kochia scoparia was slightly weaker than that of extracts from the fruits of Kochia scoparia, while the antioxidant activity of $\mathrm{N}$-trans-feruloylmethoxytyramine and N-trans-feruloyltyramine which isolated from Kochia scoparia were slightly weaker than that of vitamin $\mathrm{C}$ but better than salicylic acid. They (water, petroleum ether, chlorform,ethylacetate, and methanol) were applied by $\mathrm{Wu}$ et al [9] to extract the Fructus kochias, their fungistasis effects on six fungis were investigated by Fusarium gramine, Fusarium oxysporum, Monilia cinerea, Physalos porapiricola , Alternaria artenata and Valsa mali, respectively. The results showed that the water extracts had the strongest fungistasis effects on all six fungis, with antifungal activities all above $74.34 \%$. The five extracts indicated also stronger antifungal activities against Monilia cinerea and Valsa mali. The total flavoniod content and antioxidant activity of several extracts of Kochia scoparia fruits were analyzed by Li et al [10]. The ethanol, acetone and ethyl acetate extracts of Kochia scoparia fruits were established by cold-extraction. The total flavoid content was determined, and the DPPH assay and reducing power assay were utilized to study the antioxidant activity of Kochia scoparia fruits. All extracts of Kochia scoparia fruits possessed potent antioxidant activities, and the 
most outstanding one was found to be the ethyl acetate extract. The extracts could be utilized as natural antioxidants. Yu et al [11] determined three common treatment of urticaria $\mathrm{Fe}, \mathrm{Cu}, \mathrm{Mn}$ in traditional Chinese medicine the content of trace elements. The content of elements was obtained by flame atomic absorption spectrometry method with nitric acid and perchloric acid (4+1) decomposition samples. Three kinds of trace elements of the three kinds of traditional Chinese medicine exist obvious difference, ephedra, kochia scoparia, cortex dictamni, element content in the size are as following: $\mathrm{Fe}>\mathrm{Mn}>\mathrm{Cu}$. Zhao et al [12] investigated the effect of mycelial growth and spore germination about six kinds of aqueous extracts including Kochia scoparia , Bryophyllum pinnatum , Paeonia suffruticosa and so on by mycelial growth and spore germination method. The result indicated that there was a certain inhibition on cucumerinum, and the inhibitory activity on spore germination was better than mycelial growth. The antibacterial activity of aqueous extract of Kochia scoparia and Paeonia suffruticosa was highest.

\section{Experimental Section}

\subsection{Instruments and Reagents}

Experimental instruments: CL-1030-type high performance capillary electrophoresis (Beijing Cailu Scientific Instrument Co., Ltd.); HW2000-type chromatography workstation (Nanjing Qianpu Software Ltd.); Capillary ( $75 \mu \mathrm{m}$ inner diameter, $52 \mathrm{~cm}$ overall length, $44 \mathrm{~cm}$ effective length) from Hebei Yongnian Ruifeng Chromatographic Devices Co., Ltd.). Rutin (Shanghai Alading Biochemical Technology Co., Ltd.); Fructus Kochiae (Weifang pharmaceutical Co., Ltd.); Other reagents used in the experiments were all analytical grade; Double-distilled water was used.

\subsection{Experimental Methods}

Before the start of the experiment, capillary was successively washed with $1 \mathrm{~mol} \cdot \mathrm{L}^{-1}$ hydrochloric acid solution, double-distilled water, $1 \mathrm{~mol} \cdot \mathrm{L}^{-1}$ sodium hydroxide solution, double-distilled water, buffer solution, each for $5 \mathrm{~min}$. After three times running, capillary was cleaned again using the above method.

Measurements were carded out at $16 \mathrm{kV}$ voltage and experimental temperature at $20^{\circ} \mathrm{C}$. UV detection wavelength was $254 \mathrm{~nm}$. Injection time was $10 \mathrm{~s}$ ( $7.5 \mathrm{~cm}$ height difference).

\subsection{Sample Preparation}

Fructus Kochiae sample solution: Fructus Kochiae powder was accurately weighed 2.555 g, added $40 \mathrm{~mL}$ water with $10 \%$ methanol, extracted time of $2.5 \mathrm{~h}$ at $60^{\circ} \mathrm{C}$, filtered, washed and set the volume to $50 \mathrm{~mL}$ that was the Fructus Kochiae sample solution.

Rutin standard solution: Rutin was accurately weighed $50 \mathrm{mg}$, added $50 \mathrm{~mL}$ water with $30 \%$ methanol.

\section{Results and Discussion}

\subsection{Selection Electrophoresis Conditions}

Based on past experiment experience, we chose $20 \mathrm{mmol} / \mathrm{L}$ borax solution as a running buffer solution.

According to the literature, Rutin absorption wavelength was at $254 \mathrm{~nm}$, so we chose the $254 \mathrm{~nm}$ detection wavelength.

\subsection{Quantitative Analysis}

\subsubsection{Standard Curve}

First, Rutin standard solution that the concentration were $0.2,0.1,0.05,0.025,0.0125,0.00625$ $\mathrm{mg} / \mathrm{mL}$ was prepared. Each standard solution was run for three times under the above electrophoresis conditions and the results averaged. Taking concentration as the abscissa and peak area as the ordinate, 
the standard curve was drew. Linear regression equation of Rutin (peak area: $y \mu V \cdot s$, density: $x$ $\mathrm{mg} / \mathrm{mL})$ and the linear range was as follows: $\mathrm{Y}=3137.75+331536.12 \mathrm{X}(\mathrm{r}=0.973), 0.00625 \sim 0.2$ $\mathrm{mg} / \mathrm{mL}$.

\subsubsection{Precision Test}

A Rutin standard solution precisely drew and continuously injected for sixt times under electrophoretic separation conditions, the RSD of Rutin peak area were $8.7 \%$.

\subsubsection{Determination of Sample Content}

Under selected electrophoresis conditions, Fructus Kochiae sample solution was run. Separation chromatogram of the Fructus Kochiae sample solution was showed in Figure 1. Measured Rutin content in Fructus Kochiae was $0.115 \mathrm{mg} / \mathrm{g}(\mathrm{RSD}=10.5 \%)(\mathrm{n}=6)$.

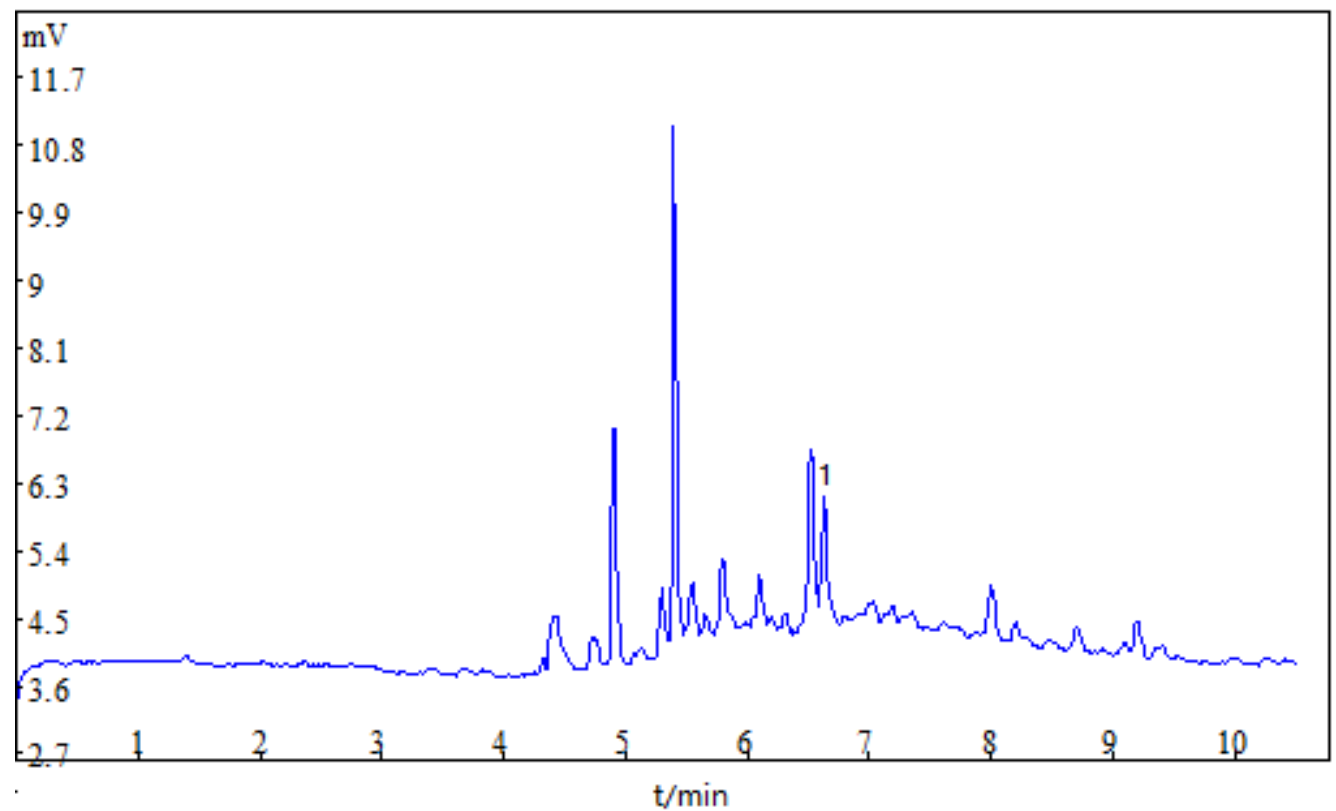

Fig. 1 Electrophorogram of Fructus Kochiae sample solution 1- Rutin

\subsubsection{Recovery}

After determination for six times, the recovery of Rutin content in Fructus Kochiae sample was $103.6 \%(\mathrm{RSD}=7.2 \%, \mathrm{n}=4)$.

\section{Acknowledgments}

This study were supported by the Natural Science Foundation of Shandong Province (No. ZR2010BL025), Open Project of State Key Laboratory of Supramolecular Structure and Materials (No. sklssm201323)(Jilin University), State Key Laboratory of Inorganic Synthesis and Preparative Chemistry (No. 2011-13)(Jilin University).

\section{References}

[1]. Chinese pharmacopoeia commission, Pharmacopoeia of the People's Republic of China(A), Beijing, China Medical Science and Technology Press, 2015: 123-124.

[2]. Lu C, Zhang HY, Ji J, Wang GX, In vivo anthelmintic activity of Dryopteris crassirhizoma, Kochia scoparia, and Polygala tenuifolia against Dactylogyrus intermedius (Monogenea)in goldfish (Carassius auratus), Parasitology research, 2012, 110(3): 1085-1090. 
[3]. Hao Wang, Chun-lin Fan, Pei Wang, et al, The study of triterpene and saponin of Fructus kochiae, Chinese Journal of Natural Medicines, 2003, 1(3): 134-136.

[4]. Xiang-hong Lu, Xiang-dong $\mathrm{Xu}$, Hong-wei $\mathrm{Fu}$, et al, The study of Fructus kochiae chemical composition, Chinese Pharmaceutical Journal, 2012, 47(5): 338-342.

[5]. De-cheng Zeng, Determination of 4'-O- $\beta$-D-glycosyl-5-O-methylvisamminol in Jingfuzhiyang Granules by RP-HPLC, Chinese Traditional Patent Medicine, 2004, 26(11): 894-897.

[6]. Guo-ping Luo, Hui-ning Meng, Cheng Chen, et al, Content determination of Momordin Ic in Fructus kochiae gel by HPLC, Science \&Technology in Chemical Industry, 2015, 23(6): 67-70.

[7]. Yu-feng Xia, Qiang Wang, Yue Dai, et al, Determination of the content of momordin Ic in kochia scoparia Fruits from different producing areas, China Journal of Chinese Materia Medica, 2002, 27(12), 890-893.

[8]. Ping Cheng, Wei Zhang, Xiu-li Peng, THE ANTIOXIDANT ACTIVITY OF KOCHIA SCOPARIA EXTRACTS AND THEIR FERULIC ACID DERIVATIVES IN VITRO, Journal of Henan University of Technology(Natural Science Edition), 2016, 37(4): 91-93.

[9]. Jing Wu, Guang-lu Shi, Xue-you Su, et al, A Preliminary Study on Bioactivity of Extracts from Fructus kochiae against several Phytopathogens, Journa of beijing university of agriculture, 2008, 23(3): 36-39.

[10]. Pei-yuan Li, Ru-mei Lu, Wei Su, et al, Total Flavoniod Content Determination and Antioxidant Activity of Kochia scoparia Friuts, Anhui Agricultural Sciences, 2016, 55(7): 10821084.

[11]. Ju-hong Yu, Rong-rong He, Jian-ming Zhao, et al, Three kinds of treatment of urticaria TCM fe, $\mathrm{cu}, \mathrm{mn}$ determination of trace elements, Studies of Trace Elements and Health, 2014, 31(2): $32-33$.

[12]. Cheng-ai Zhao, Hui Sun, Cheng-yu Wang, et al, Inhibition Screening and Activity of 6 Kinds of Chinese Herbal Medicines on Cladosporium cucumerinum, Agrochemicals, 2010, 49(6): 453455. 